\section{The Relationship Between Emotion Recognition from Facial Expression and Self-Construal}

\author{
Shinnosuke Ikeda* \\ Kyoto University of Advanced Science, 18 Gotanda-cho, Yamanouchi, \\ Ukyo-ku, Kyoto, 615-8577, Japan \\ "Author for correspondence (odenshin@gmail.com)
}

Previous studies have identified cultural differences in the patterns of emotion recognition, with Eastern cultures emphasizing emotional expression through the eyes and Western cultures emphasizing the facial expression around the mouth. The influence of selfconstrual has been considered a factor of these cultural differences, but no direct examination has been conducted to clarify how cultural factors are related to self-construal. To examine this relationship and exclude cultural influences other than relational self-construal, this study involved three experiments regarding self-construal and emotion recognition in Japanese subjects. The results showed that the participants perceive sadness more strongly through the eye region when they have a high degree of interdependence, and they perceive happiness more strongly through the mouth region when they have a high degree of independence. The results partially confirm the results of previous research and highlight that self-construal plays a role in interpreting facial cues; these findings suggest that more detailed studies and research focusing on other cultures should be conducted to clarify (1) cultural influence on self-construal and (2) cultural influence on emotion recognition.

\section{Keywords}

emotion recognition, facial expression, facial cues, selfconstrual, emotion, Japanese

\section{Introduction}

Facial expressions help us gauge others' emotions (e.g., Jack \& Schyns, 2015). While expressions have an evolutional basis (Jack et al., 2014) and are somewhat culture-universal (Ekman, 1992), they also have socioculturally constructed culture-specific characteristics (Elfenbein et al., 2007; Marsh et al., 2003), and people most readily recognize the emotions of others within their own culture (Elfenbein \& Ambady, 2002, 2003).

What are the differences in facial expressions among cultures that are not evolutionarily acquired? One involves the facial regions emphasized in facial expressions. Jack et al. (2012) examined the internal representations of six facial expressions, including joy, anger, and sadness, in Westerners (Europeans and North Americans) and Easterners (Chinese). They found that Westerners' facial expressions mostly involve the eyebrows and mouth, while Easterners' are mostly characterized by their eyes.

These cultural characteristics of facial expressions also affect viewers' gaze-focus patterns in recognizing emotions from facial expressions. Jack et al. (2009) reported that Westerners (Europeans) gaze at the entire face, while Easterners (Chinese and Japanese) gaze specifically at the eyes to recognize emotions from facial expressions. Yuki et al. (2007) also examined which cue was more important for Westerners (Americans) and Easterners (Japanese), presenting subjects with facial expressions where the eyes and mouth expressed different emotions and asking them to rate the emotions of the expresser. The results showed eyes as more important to Easterners and the mouth as more important to Westerners for interpreting the expresser's emotion.

However, previous studies have not clarified what factors explain these cultural differences. Yamamoto et al. (2020) argued that these differences might be caused by broader cultural frameworks such as self-construal (Markus \& Kitayama, 1991) rather than cultural aspects that differ across countries. Yamamoto et al. (2020) also reported results similar to Yuki et al. (2007), examining Japanese and Dutch subjects' interpretations of emoticons whose eyes expressed different emotions than the mouths. Results obtained by comparing Japan and the U.S. (Yuki et al., 2007) may also be explained by self-construal differences related to the broader cultural framework (cf., Tanaka et al., 2010).

Self-construal is a constructive concept that indicates how the self is perceived in relation to others in each culture; Easterners have a stronger sense of interdependence while Westerners have a stronger sense of independence (Markus \& Kitayama, 1991). Factors of self-construal's evolutionary origins, such as geography, agriculture, and risk of pathogen infection, have been discussed (Chiao \& Blizinsky, 2010; Kitayama et al., 2006; Talhelm et al., 2014). Human cognitive systems are thought to have co-evolved with these factors (Richerson \& Boyd, 2005), as self- and other-cognition, motivation, and emotions are acquired to adapt (Markus \& Kitayama, 1991). For example, it has been suggested that pressures to suppress emotional expression are relatively stronger in Eastern cultures that more highly value interdependence (compared to Western cultures) (Matsumoto et al., 1998; Murata et al., 2013). Considering that the eyes are more difficult to manipulate intentionally than the mouthand expressions around the eyes are harder to suppress(Ekman et al., 1988), the eyes may be the facial aspect that reveals the true emotions of others. Expressions around the mouth are the most conspicuous; hence, the mouth may be 
more revealing for Westerners because their feelings are less likely to be suppressed (cf., Ozono et al., 2010).

This study elucidates the factors that explain the cultural differences in the tendency to emphasize eyes and mouth in recognizing emotions from facial expressions. Therefore, as Yamamoto et al. (2020) suggested, the present study focused on Japanese participants' selfconstrual-measured via questionnaire - and the cultural emphasis on the eyes or mouth for interpreting emotions from facial expressions. The present study included only participants from Japan to exclude cultural factors other than self-construal. It was predicted that higher interdependence would be correlated with more emphasis on the eyes, and higher independence would be correlated with more emphasis on the mouth for recognizing emotions based on facial expressions.

\section{Experiment 1}

Methods

(a) Participants

Fifty-four Japanese university students (mean age $=20.2$ years, $S D=4.27,35$ females) attending a university in the Kanto region of Japan participated in the experiment. Students participated in the experiment as part of their psychology major classes but were free to choose whether to participate. Written informed consent was obtained from all participants.

\section{(b) Materials}

As standardized facial expression stimuli, both the happy and the sad facial expressions of four women from the ATR facial expression database (http://www.atr-p.com/ products/face-db.html) were selected. The eye regions of each expression were cut out and pasted onto the eyes of the other emotional expressions by the same person to create photographs of facial expressions with eyes and mouths representing differing emotions. Four types of facial expressions were prepared: happy, sad, happy eyes/ sad mouth, and sad eyes/happy mouth. There were 16 total facial expressions (four facial expressions by each of the four females). Examples of facial expressions are shown in Figure 1.

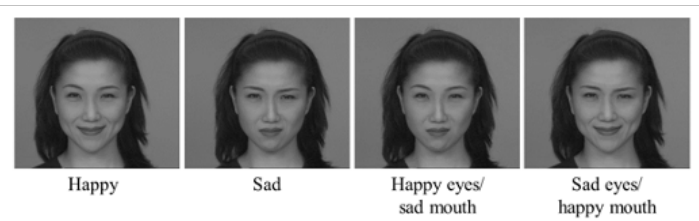

Figure 1. Examples of the facial expressions used in Experiment 1 .

This study used the Revised Independent and Interdependent Self-Construal Scale (Takata et al., 1996) to measure participants' internal sense of interdependence and independence ${ }^{1}$. This questionnaire consists of 20 items (10 interdependence items; 10 independence items), each scored on a 7-point scale.

${ }^{1}$ In this study, self-construal was measured using the questionnaire because of its methodological simplicity. However, it is necessary to pay attention to the validity of such an explicit method of measuring self-construal (Kitayama et al., 2009).

\section{(c) Procedure}

The experiment was conducted face-to-face. Self-construal can be manipulated by priming (Brewer \& Gardner, 1996). Therefore, to prevent the emotion recognition procedure from affecting their self-construal, the participants first answered the Revised Independent and Interdependent Self-Construal Scale (Takata et al., 1996) and then individually participated in an emotion recognition task.

In the emotion recognition task, one happy expression and one sad expression by a male, which were not used in the main trial, were presented as practice trials. In the main trial, 16 facial expressions were presented one by one. Participants were asked to rate the expressions on a 9-point scale from "extremely sad" to "extremely happy." The stimuli were presented on the screen by PsychoPy ver.1.82 (Peirce, 2007); responses were counted using the keyboard's numeric keys. The order of facial expressions in the practice and main trials were randomized.

\section{(d) Scoring}

The self-construal-scores were calculated as the mean of the responses to the items measuring interdependence and independence. The higher the score, the stronger the self-construal. In the emotion recognition task, the mean ratings for the four types of facial expressions were calculated on a 9-point scale, with higher values indicating the participants' "happier" interpretations and lower values indicating "sadder" ratings.

\section{Results}

The means of the ratings for each facial expression and self-construal-score are shown in Table 1. The results of the comparison of the expression ratings are shown in the Supplemental Information.

Next, multiple regression analysis was conducted for the four types of facial expressions separately (independent variables: interdependence and independence; dependent variable: the rating value). No significant effects of self-construal scores were found for any of the facial expressions (Table 2).

\section{Discussion}

Experiment 1 examined the relationship between emotion recognition and self-construal. Contrary to the initial prediction, there was no relationship between interdependence and the eye-focus tendency or between independence and the mouth-focus tendency. This result is contrary to Yamamoto et al.'s (2020) suggestion that selfconstrual determines which cues are important.

However, although the design of Experiment 1 was based on Yuki et al. (2007), it was inadequate for examining the relationship between self-construal and emotion recognition because the scale had "extremely sad" and "extremely happy" at the extremes. Therefore, for example, if both sad and happy emotions are recognized in the sad eyes/happy mouth expression, even if sadness is seen strongly in the eyes, it may be affected by the happiness expressed by the mouth, and the rating may not reflect emotion recognition from the eyes or mouth region appropriately. Therefore, Experiments 2 and 3 were conducted using happy and sad facial expressions combined with the neutral expression so that only the eyes or mouth expressed the emotion. For both experiments, it 
Table 1. Mean and $S D$ of each score in Experiment 1.

\begin{tabular}{lcccccc}
\hline & Interdependence & Independence & Happy & $\begin{array}{c}\text { Happy eyes/ } \\
\text { Sad mouth }\end{array}$ & $\begin{array}{c}\text { Sad eyes/ } \\
\text { Happy mouth }\end{array}$ & Sad \\
\hline Mean & 5.02 & 4.40 & 6.64 & 4.14 & 4.65 & 2.80 \\
$S D$ & $(0.85)$ & $(1.00)$ & $(0.74)$ & $(0.69)$ & $(0.89)$ & $(0.58)$ \\
\hline
\end{tabular}

Table 2. Results of multiple regression analysis in Experiment 1.

\begin{tabular}{lccccccccc}
\hline & \multicolumn{1}{c}{ Happy } & \multicolumn{4}{c}{ Happy eyes/ } & \multicolumn{2}{c}{ Sad eyes/ } & \multicolumn{2}{c}{ Sad } \\
& $\beta$ & $p$ & $\beta$ & $p$ & $\beta$ & $p$ & $\beta$ & $p$ \\
\hline Interdependence & -0.003 & .806 & -0.015 & .242 & 0.005 & .759 & 0.005 & .624 \\
Independence & 0.008 & .496 & -0.007 & .490 & 0.025 & .074 & -0.002 & .762 \\
\hline $\mathrm{R}^{2}$ & .017 & .646 & .027 & .494 & .068 & .167 & .118 & .739 \\
\hline
\end{tabular}

was predicted that the eyes would be more important for those with high interdependence, and the mouth would be more important for those with high independence.

\section{Experiment 2}

Methods

(a) Participants

Twenty-five Japanese university students (mean age $=$ 19.3 years, $S D=0.69,16$ females) who did not participate in Experiment 1 participated in this experiment. The conditions were the same as in Experiment 1.

\section{(b) Materials}

The same procedure as in Experiment 1 was used to synthesize happy and neutral expressions. A total of 16 images of four types of expressions-happy, neutral, happy eyes/neutral mouth, and neutral eyes/happy mouth - were prepared. Examples of facial expressions are shown in Figure 2.

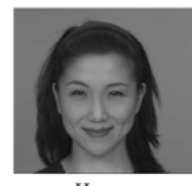

Happy

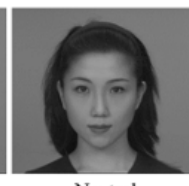

Neutral

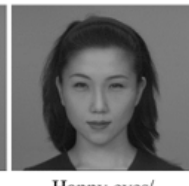

Happy eyes' Happy eyes
neutral mouth

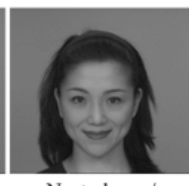

Neutral eyes' happy mouth
Figure 2. Examples of the facial expressions in Experiment 2.

\section{(c) Procedure}

The procedure was the same as in Experiment 1. In the emotion recognition task, the participants were asked to rate the expressions using a 5-point scale from "neutral" to "extremely happy." The 5-point method was adopted because Experiment 1 used a 9-point scale ranging from "extremely sad" to "extremely happy," and the intermediate value of this 9-point scale was set as "neutral" to correspond to the 5-point scale in Experiment 2.

\section{(d) Scoring}

The self-construal score and performance on the emotion recognition task were scored using the same procedure as in Experiment 1. Each facial expression was rated on a 5-point scale, with a higher score indicating that the subject perceived the expression as "happier."

\section{Results}

The means of the ratings and cultural self-view scores for each facial expression are shown in Table 3. The results of the comparison of the expression ratings are shown in the Supplemental Information.

Multiple regression analysis was conducted for the four types of facial expressions (independent variables: interdependence and independence: dependent variable: the rating). As a result, independence scores significantly predicted neutral eyes/happy mouth ratings (Table 4).

\section{Discussion}

Experiment 2 examined the relationship between emotion recognition from the eyes and mouth and self-construal by synthesizing happy and neutral faces. A significant positive influence was found between independence and the neutral eyes/happy mouth rating: the higher the independence, the stronger the recognition of happiness through the mouth. Thus, the prediction was partially supported.

\section{Experiment 3}

Methods

\section{(a) Participants}

Twenty-four Japanese university students (mean age $=19.8$ years, $S D=2.06,16$ females) who had not participated in Experiments 1 and 2 participated in the experiment. The conditions were the same as in Experiment 1 .

\section{(b) Materials}

The same procedure as in Experiment 2 was used to synthesize sad and neutral facial expressions. Four types of image expressions were prepared: sad, neutral, sad eyes/ neutral mouth, and neutral eyes/sad mouth. Examples of facial expressions are shown in Figure 3.

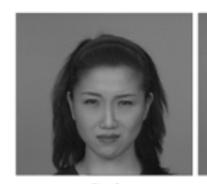

Sad

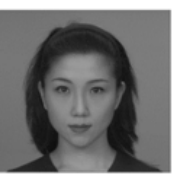

Neutral

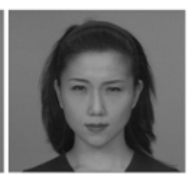

Sad eyes' neutral mouth

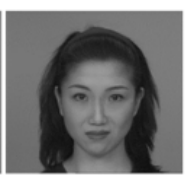

Neutral eyes'
Figure 3. Examples of the facial expressions used in Experiment 3 . 
Table 3. Mean and $S D$ of each score in Experiment 2.

\begin{tabular}{lcccccc}
\hline & Interdependence & Independence & Happy & $\begin{array}{c}\text { Happy eyes/ } \\
\text { neutral mouth }\end{array}$ & $\begin{array}{c}\text { Neutral eyes/ } \\
\text { happy mouth }\end{array}$ & Neutral \\
\hline Mean & 5.00 & 4.40 & 3.56 & 1.45 & 3.06 & 1.27 \\
$S D$ & $(0.57)$ & $(0.90)$ & $(0.66)$ & $(0.45)$ & $(0.54)$ & $(0.34)$ \\
\hline
\end{tabular}

Table 4. Results of multiple regression analysis in Experiment 2.

\begin{tabular}{lccccccccc}
\hline & \multicolumn{2}{c}{ Happy } & \multicolumn{4}{c}{ Happy eyes/ } & \multicolumn{2}{c}{ Neutral eyes/ } & \multicolumn{2}{c}{ Neutral } \\
& $\beta$ & $p$ & $\beta$ & $p$ & $\beta$ & $p$ & \multicolumn{1}{c}{$\beta$} & $p$ \\
\hline Interdeppendence & 0.016 & .539 & 0.016 & .352 & 0.003 & .873 & 0.012 & .340 \\
Independence & 0.015 & .340 & 0.001 & .919 & $0.031^{*}$ & .012 & -0.008 & .303 \\
\hline $\mathrm{R}^{2}$ & .047 & .591 & .041 & .632 & $.263^{*}$ & .035 & .117 & .256 \\
\hline$* p<.05$ & & & & & & & & &
\end{tabular}

\section{(c) Procedure}

The procedure was the same as in Experiment 1. In the emotion recognition task, the participants were asked to rate the expressions using a 5-point scale from "neutral" to "extremely sad."

\section{(d) Scoring}

The self-construal scores and performance on the emotion recognition task were scored using the same procedure as in Experiment 2.

\section{Results}

The self-construal scores and the means of each facial expression's rating are shown in Table 5. The results of the comparison of the expression ratings are shown in the Supplemental Information.

Multiple regression analysis was conducted for the four types of facial expressions (independent variables: interdependence and independence; dependent variable: the rating). The results showed that interdependence significantly predicted the sad and sad eyes/neutral mouth ratings (Table 6).

\section{Discussion}

Experiment 3 examined the relationship between emotion recognition from the eyes and mouth and self-construal by synthesizing sad and neutral faces. A significant positive influence was found: the higher the interdependence, the stronger the perception of sadness from the sad facial expression, and the stronger the perception of sadness from the eyes. As in Experiment 2, the prediction was partially supported.

\section{General Discussion}

This study examined the relationship between selfconstrual and tendencies in emotion recognition from facial expressions where eyes and mouth present differing emotions. The results showed that the higher the independence, the stronger the happiness recognized from the mouth-only happy facial expressions, and the higher the interdependence, the stronger the sadness recognized from the eyes-only sad facial expressions. There was no general tendency to emphasize the mouth more when independence was high, or the eyes more when interdependence was high; the predictions were only partially supported for specific emotions.

Why was there such an association between selfconstrual and emotion recognition only for specific emotions? During the course of evolution, humans acquired a process of emotion recognition that consists of two processes (Jack et al., 2014). First, humans quickly recognize whether one should approach or avoid others. After that, emotions are recognized based on categories such as "happiness" and "sadness," taking the context into account. It has been suggested that this latter processing is

Table 5. Mean and $S D$ of each score in Experiment 3.

\begin{tabular}{|c|c|c|c|c|c|c|}
\hline & Interdependence & Independence & $\mathrm{Sad}$ & $\begin{array}{l}\text { Sad eyes/neutral } \\
\text { mouth }\end{array}$ & $\begin{array}{l}\text { Neutral eyes/ } \\
\text { sad mouth }\end{array}$ & Neutral \\
\hline Mean & 5.29 & 4.38 & 3.93 & 2.94 & 2.06 & 1.22 \\
\hline$S D$ & $(0.53)$ & $(0.83)$ & $(0.61)$ & $(0.76)$ & $(0.57)$ & $(0.49)$ \\
\hline
\end{tabular}

Table 6. Results of multiple regression analysis in Experiment 3.

\begin{tabular}{lccccccccc}
\hline & \multicolumn{2}{c}{ Sad } & \multicolumn{2}{c}{ Sad eyes/ } & \multicolumn{2}{c}{ Neutral eyes/ } & \multicolumn{2}{c}{ Neutral } \\
& $\beta$ & $p$ & $\beta$ & $p$ & $\beta$ & $p$ & $\beta$ & $p$ \\
\hline interdependence & $0.038^{*}$ & .037 & $0.064^{* *}$ & .003 & 0.030 & .099 & 0.008 & .592 \\
independence & 0.024 & .130 & 0.035 & .059 & 0.006 & .686 & 0.009 & .515 \\
\hline $\mathrm{R}^{2}$ & .211 & .084 & $.355^{*}$ & .010 & .127 & .241 & .025 & .766 \\
\hline$* p<.05, * * p<.01$ & & & & & & & &
\end{tabular}


shaped by sociocultural norms (Gendron et al., 2016). This allows for the development of adaptive emotion recognition in cultures where interdependency or independency predominate. The results of this study may reflect such acquired emotional recognition. Additionally, perhaps because the importance of expressing certain emotions differs between interdependent and independent selfconstrual. In Eastern cultures, the ability to detect sadness and the inclination to help others were, presumably, significant in the process of interdependency prevailing, due to reasons such as geography and agriculture (Ikeda, 2020; Uchida et al., 2008). Contrastingly, in Western cultures, the need for autonomy, and the experience and expression of emotions such as happiness, were probably important in the process of independency prevailing (Eid \& Diener, 2001; Kitayama et al., 2006).

Participants from Japan's higher degree of interdependence reflects that more value is placed on harmony and sensitivity to the negative expressions of others; therefore, more importance is placed on the eyes, which are difficult to manipulate intentionally, especially in sad expressions. In contrast, a higher degree of independence means more importance is placed on the expression of happy emotions, and, thus, more importance is placed on the mouth where emotions can be read clearly (Calder et al., 2000). These individual differences in emotion recognition may go beyond evolutionary commonalities as people adapt communication respective to social cultures.

Additionally, a series of experiments have shown that Japanese people do not always focus only on the eyes to read emotion; they focus on the mouth when expressing happiness and on the eyes when expressing sadness (Supplemental Information). Similar results were found in a study of Westerners. Japanese people only place relative importance on the eyes compared to Westerners, but, in absolute terms, they place importance on the mouth as well, depending on the emotion, just as Westerners do.

This study examined the relationship between selfconstrual and emotion recognition in a Japanese sample. The results show that the predictions were partially supported only for specific emotions. Therefore, it is necessary to examine the relationship between selfconstrual and emotion recognition patterns by measuring self-construal rather than relying on cultural comparison study results (e.g., Yamamoto et al., 2020; Yuki et al., 2007). Patterns might be seen depending on the emotion; therefore, it is necessary to check the details of the relationship between self-construal and emotion recognition by using facial expressions with only the eyes or only the mouth expressing emotion, and examining various emotions (e.g., happiness, sadness, anger, fear). Although the results of this study were consistent with previous studies, the sample size was relatively small, so it is necessary to examine the aforementioned issues with a larger sample in the future. In the future, it is hoped that the evolutionary formation of these cultural characteristics will be further elucidated.

\section{Acknowledgement}

This work was supported by JSPS KAKENHI (20K22295). This study was approved by Ethics Committee of Kyoto
University of Advanced Science (\#20-506).

\section{Supplementary Material}

Electronic supplementary material is available online.

\section{References}

Brewer, M. B., \& Gardner, W. (1996). Who is this "We"? Levels of collective identity and self representations. Journal of Personality and Social Psychology, 71(1), 83-93. https://doi.org/10.1037/0022-3514.71.1.83

Calder, A. J., Young, A. W., Keane, J., \& Dean, M. (2000). Configural information in facial expression perception. Journal of Experimental Psychology: Human Perception and Performance, 26(2), 527-551. https://doi.org/10.1037//0096-1523.26.2.527

Chiao, J. Y., \& Blizinsky, K. D. (2010). Culture-gene coevolution of individualism-collectivism and the serotonin transporter gene. Proceedings of the Royal Society B: Biological Sciences, 277(1681), 529-537. https://doi.org/10.1098/rspb.2009.1650

Eid, M., \& Diener, E. (2001). Norms for experiencing emotions in different cultures: Inter- and intranational differences. Journal of Personality and Social Psychology, 81(5), 869-885. https://doi. org/10.1037/0022-3514.81.5.869

Ekman, P. (1992). An argument for basic emotions. Cognition and Emotion, 6(3-4), 169-200. https://doi. org/10.1080/02699939208411068

Ekman, P., \& Friesen, W. V. (1975). Unmasking the face: A guide to recognizing emotions from facial expressions. Prentice Hall.

Ekman, P., Friesen, W. V., \& O’Sullivan, M. (1988). Smiles when lying. Journal of Personality and Social Psychology, 54(3), 414-420. https://doi. org/10.1037/0022-3514.54.3.414

Elfenbein, H. A., \& Ambady, N. (2002). Is there an in-group advantage in emotion recognition? Psychological Bulletin, 128(2), 243-249. https://doi. org/10.1037/0033-2909.128.2.243

Elfenbein, H. A., \& Ambady, N. (2003). Universals and cultural differences in recognizing emotions. Current Directions in Psychological Science, 12(5), 159-164. https://doi.org/10.1111/1467-8721.01252

Elfenbein, H. A., Beaupré, M., Lévesque, M., \& Hess, U. (2007). Toward a dialect theory: Cultural differences in the expression and recognition of posed facial expressions. Emotion, 7(1), 131-146. https://doi. org/10.1037/1528-3542.7.1.131

Ikeda, S. (2020). Social anxiety enhances sensitivity to negative transition and eye region of facial expression. Personality and Individual Differences, 163(1). https:// doi.org/10.1016/j.paid.2020.110096

Gendron, M., Roberson, D., van der Vyver, J. M., \& Barrett, L. F. (2014). Perceptions of emotion from facial expressions are not culturally universal: Evidence from remote culture. Emotion, 14(2), 251262. https://doi.org/10.1037/a0036052.Perceptions

Jack, R. E., Blais, C., Scheepers, C., Schyns, P. G., \& Caldara, R. (2009). Cultural confusions show that facial expressions are not universal. Current Biology, 19(18), 1543-1548. https://doi.org/10.1016/ j.cub.2009.07.051

Jack, R. E., Caldara, R., \& Schyns, P. G. (2012). Internal representations reveal cultural diversity in expectations of facial expressions of emotion. Journal 
of Experimental Psychology: General, 141(1), 19-25. https://doi.org/10.1037/a0023463

Jack, R. E., Garrod, O. G. B., \& Schyns, P. G. (2014). Dynamic facial expressions of emotion transmit an evolving hierarchy of signals over time. Current Biology, 24(2), 187-192. https://doi.org/10.1016/ j.cub.2013.11.064

Jack, R. E., \& Schyns, P. G. (2015). The human face as a dynamic tool for social communication. Current Biology, 25(14), R621-R634. https://doi.org/10.1016/ j.cub.2015.05.052

Kitayama, S., Ishii, K., Imada, T., Takemura, K., \& Ramaswamy, J. (2006). Voluntary settlement and the spirit of independence: Evidence from Japan's "northern frontier." Journal of Personality and Social Psychology, 91(3), 369-384. https://doi. org/10.1037/0022-3514.91.3.369

Kitayama, S., Park, H., Sevincer, A. T., Karasawa, M., \& Uskul, A. K. (2009). A cultural task analysis of implicit independence: Comparing North America, Western Europe, and East Asia. Journal of Personality and Social Psychology, 97(2), 236-255. https://doi. org/10.1037/a0015999

Markus, H. R., \& Kitayama, S. (1991). Culture and the self: Implications for cognition, emotion, and motivation. Psychological Review, 98(2), 224-253. https://doi. org/10.1037/0033-295X.98.2.224

Marsh, A. A., Elfenbein, H. A., \& Ambady, N. (2003). Non-verbal "Accents": Cultural differences in facial expressions of emotion. Psychological Science, 14(4), 373-376. https://doi.org/10.1111/1467-9280.24461

Matsumoto, D., Takeuchi, S., Andayani, S., Kouznetsova, N., \& Krupp, D. (1998). The contribution of individualism vs. collectivism to cross-national differences in display rules. Asian Journal of Social Psychology, 1(2), 147-165. https://doi. org/10.1111/1467-839X.00010

Murata, A., Moser, J. S., \& Kitayama, S. (2013). Culture shapes electrocortical responses during emotion suppression. Social Cognitive and Affective Neuroscience, 8(5), 595-601. https://doi.org/10.1093/ scan/nss036

Ozono, H., Watabe, M., Yoshikawa, S., Nakashima, S., Rule, N. O., Ambady, N., \& Adams, R. B. (2010). What's in a smile? Cultural differences in the effects of smiling on judgments of trustworthiness. Letters on Evolutionary Behavioral Science, 1(1), 15-18. https:// doi.org/10.5178/lebs.2010.4

Peirce, J. W. (2007). PsychoPy-Psychophysics software in Python. Journal of Neuroscience Methods, 162(1-2), 8-13. https://doi.org/10.1016/j.jneumeth.2006.11.017

Richerson, P. J., \& Boyd, R. (2005). Not by genes alone: How culture transformed human evolution. University of Chicago Press. https://doi.org/10.7208/ chicago/9780226712130.001.0001

Takata, T., Omoto, M., \& Seike, M. (1996). Construction of a revised scale for independent and interdependent construal of self. Memoirs of the Nara University, 24, 157-173. http://repo.nara-u.ac.jp/modules/ xoonips/detail.php?id=AN00181569-19960300-1012 (ISSN)03892204

Talhelm, T., Zhang, X., Oishi, S., Shimin, C., Duan, D., Lan, X., \& Kitayama, S. (2014). Large-scale psychological differences within China explained by rice versus wheat agriculture. Science, 344(6184), 603-608. https://doi.org/10.1126/science.1246850

Tanaka, A., Koizumi, A., Imai, H., Hiramatsu, S.,
Hiramoto, E., \& de Gelder, B. (2010). I feel your voice: Cultural differences in the multisensory perception of emotion. Psychological Science, 21(9), 1259-1262. https://doi.org/10.1177/0956797610380698

Uchida, Y., Kitayama, S., Mesquita, B., Reyes, J. A. S., \& Morling, B. (2008). Is perceived emotional support beneficial? Well-being and health in independent and interdependent cultures. Personality and Social Psychology Bulletin, 34(6), 741-754. https://doi. org/10.1177/0146167208315157

Yamamoto, H. W., Kawahara, M., Kret, M. E., \& Tanaka, A. (2020). Cultural differences in emoticon perception: Japanese see the eyes and Dutch the mouth of emoticons. Letters on Evolutionary Behavioral Science, 11(2), 40-45. https://doi.org/10.5178/ lebs. 2020.80

Yuki, M., Maddux, W. W., \& Masuda, T. (2007). Are the windows to the soul the same in the East and West? Cultural differences in using the eyes and mouth as cues to recognize emotions in Japan and the United States. Journal of Experimental Social Psychology, 43(2), 303-311. https://doi.org/10.1016/ j.jesp.2006.02.004 